\title{
Important Recent Advances in Gynaecological Pathology
}

\author{
Sanjiv Manek \\ Consultant Gynaecological Pathologist \\ Oxford, UK
}

In recent years there have been a significant number of changes in gynaecological pathology practice. Many of these deal with the molecular basis of common and rare gynaecological tumours but there are also many that deal with morphology, terminology and new perspectives. This talk will focus on a few chosen entities and concepts that have been recently introduced and have reached everyday practice but which can cause problems or diagnostic difficulties and can require alteration in protocols.

The areas to be covered are:

The concept of endometrial intraepithelial neoplasia (EIN)

The concepts of endometrial intraepithelial carcinoma (EIC) and minimal serous carcinoma

Undifferentiated endometrial carcinoma

Inherited endometrial tumour syndromes

The relevance of tubal carcinoma in situ and the preceding dysplastic lesion in the pathogenesis of pelvic serous carcinoma

The concept of differentiated VIN and its association with squamous carcinoma

Microinvasion in borderline serous ovarian tumours

The implications of the 2009 FIGO staging system for endometrial and vulval malignancies and uterine sarcomas

\section{Endometrial intraepithelial neoplasia (EIN)}

The 2003 WHO and ISGP review of endometrial hyperplasia has retained the following classification.

Hyperplasias (typical)

Simple without atypia

Atypical Hyperplasia

Complex without atypia

Simple atypical

Complex atypical

The atypia refers to cytological rather than architectural atypia and is commonly diagnosed when the nuclear/cytoplasmic ratio is increased and there is loss of axial polarity with nuclei becoming large and rounded with prominent nucleoli. Mitoses and apoptotic bodies are frequently found.

Because of the common problem of reproducibility in identifying genuine cytological atypia, an alternative classification, the endometrial intraepithelial neoplasia (EIN) system has been proposed and is being increasingly used. In this system, there is a spectrum from benign hyperplasias through EIN to adenocarcinoma. The EIN approach is supported by morphometric, molecular and genetic data. There is a concept of latency with PTEN mutation. Some latent glands may involute and others may progress to EIN. From the molecular point of view, EIN is a monoclonal proliferation with PTEN mutation and microsatellite instability.

Histologically, EIN is identified by:

Foci of atypical hyperplasia at least $1 \mathrm{~mm}$ and greater in linear dimension (usually $>5-10$ gland clusters).

Gland to stroma ratio $>1$.

Cytological features that are different from those of background glands.

Exclusion of potential mimics such as disordered proliferation, polyps and adenocarcinomas.

Most complex atypical hyperplasias could be reclassified as EIN.

In carcinoma cases with background atypical hyperplasia/EIN, PTEN mutations, b-Catenin, MLH 1 and $\mathrm{K}$-ras oncogene mutations and microsatellite instability are seen in both processes, lending support to the fact that atypical hyperplasia/EIN is pre-cancerous. Many studies have shown an increased risk of associated 
carcinoma when an endometrial biopsy shows atypical hyperplasia. With EIN this risk is up to 46x more than without EIN.

It may be difficult to distinguish between atypical hyperplasia/EIN and well-differentiated (FIGO Grade

1) endometrial adenocarcinoma. Features which help to identify adenocarcinoma are:

prominent nuclear pleomorphism

a confluent, fused glandular pattern

a cribriform architecture of glands

a confluent papillary pattern

desmoplastic stroma

replacement of stroma by squamous epithelium

The confluent or papillary pattern or squamous epithelial predominance has to occupy at least half of a low-power field.

\section{$\underline{\text { EIC and minimal serous carcinoma }}$}

Endometrial intraepithelial carcinoma (EIC) or serous EIC is the precursor lesion of serous endometrial adenocarcinoma and occurs typically in post-menopausal women on a background of atrophy. It comprises highly pleomorphic cells on the surface and in glands without invasion but with frequent mitoses and prominent nucleoli in atypical nuclei. Mib 1 (Ki67) staining shows a high proliferation index and there is strong p53 staining indicating p53 gene mutation. Rarely, there may be microinvasion associated with EIC. It is well known that EICs can be associated with a high incidence of extrauterine metastatic disease despite lack of invasion in the uterus. Hence, the concept of 'minimal uterine serous carcinoma' has developed. This is diagnosed when there is EIC with focal superficial/surface serous carcinoma. These lesions are WT1 and ER negative. Recently, it has been shown that some serous carcinomas are WT1+ and p53 - (?dedifferentiation of type I cancers). Another lesion, endometrial glandular dysplasia (EmGD) has been described and this appears to be a precursor of EIC.

\section{Undifferentiated endometrial carcinoma}

This is a new entity which is now increasingly used but it is poorly recognised because of its 'weak definition' in the literature. It is generally included in FIGO G3 endometrioid adenocarcinomas in textbooks. The WHO definition is 'no evidence of glandular or squamoid differentiation '. The tumour is composed of medium or large cells with a complete absence of glandular differentiation and absent or minimal ( < $10 \%)$ neuroendocrine differentiation. There are no specific gross features and microscopically there are solid sheets of these medium or large cells with marked pleomorphism, rhabdoid cells, foci of necrosis and many mitoses ( $>10 / 10 \mathrm{hpf})$. Immunohistochemically, they are CK focally positive, EMA positive, Vimentin positive and $<10 \%$ cells show 1 positive neuroendocrine marker. Its significance is in that it has a poor prognosis $(75 \%$ cases died of disease), as compared to the excellent prognosis of G2 and intermediate prognosis of G3 endometrioid carcinomas ( $30 \%$ cases died of disease)

\section{Inherited endometrial tumour syndromes}

Lynch Syndrome or Hereditary Nonpolyposis Colon Cancer Syndrome (HNPCC) patients are vulnerable to developing endometrioid adenocarcinomas and this may be the initial presentation in more than $50 \%$ of cases. They usually occur in young pre-menopausal patients and are more common than colorectal cancers in female patients with this syndrome. The genetic defect is in DNA mismatch repair genes (MLH-1, MSH6 and

14. Kongres udruženja patologa i citologa Srbije sa međunarodnim učešćem, Beograd 14-16 juna, 2012

14 th Congress of Serbian Association of Pathologists and Cytologists with international participation, Belgrade 14-16 June, 2012 
MSH2). Women with HNPCC have a 20\% chance of developing endometrial cancer compared to 3\% without HNPCC. HNPCC/Lynch Syndrome endometrial cancers have the following characteristics:

predilection for isthmic and cornual areas

poor differentiation

peritumoral, Crohn's-like lymphoid reaction

intratumoral lymphocytic infiltration

higher incidence of lymphatic permeation

higher incidence of mixed carcinomas and carcinosarcomas

Tubal carcinoma in-situ (serous tubal intraepithelial carcinoma - STIC) and its precursor lesions and the link to pelvic serous carcinoma

There is a vast amount of new data showing the increasing importance of STIC. In a significant number of cases of endometrial serous carcinoma, including EIC, there is co-existent STIC with similar p53 signatures. In some cases, the STIC is WT1 negative suggesting spread from the endometrium or at least a field change. In other cases the STIC is WT1 positive indicating a synchronous lesion when the endometrial primary is WT1 negative. STIC or tubal invasive serous carcinoma may also be the origin of some endometrial serous carcinomas which have the reversed immunoprofile of WT1 positivity and p53 negativity.

The STIC is also thought to be the origin of many peritoneal and ovarian serous carcinomas particularly in cases of high grade serous histology. Until recently it was suggested that the p53 signature (synonymous with tubal dysplasia and the tubal intraepithelial lesions in transition - TILTs) was only limited to BRCA+ cases but new evidence shows that its importance goes beyond that.

\section{Vulval intraepithelial neoplasia (VIN) and squamous carcinoma (SCC)}

There are two distinct types of VIN; each with a related SCC:

\begin{tabular}{l} 
Classical (Bowenoid) \\
1. Undifferentiated appearance \\
Basaloid $\leftrightarrow$ Warty \\
2. Associated with high risk HPV $(16,18)$ \\
3. Multifocal \\
4. \pm CIN, VAIN, warts \\
5. Young women \\
6. Lower risk for SCC \\
7. p16 positive \\
8. p53 negative \\
9. Associated with basaloid/warty/ verrucous SCC \\
10. Rare mucinous differentiation \\
\hline
\end{tabular}

\section{Differentiated (Simplex)}

1. Difficult to diagnose in isolation

(i.e. without adjacent SCC)

2. Hyperplastic epithelium with basal atypia

3. Arises in vulval dystrophy (e.g hyperplasia) or lichen sclerosus

4. Older women

5. p16 negative

6. p53 positive

7. Associated with keratinising SCC

8. Basaloid variant recognised

Recently, there has been a proposal to reclassify VIN. Essentially it has been suggested that in classic VIN, the VIN 1 category be abandoned because of its rarity and instead be referred to as flat condyloma or HPV effect and VIN 2 and VIN 3 be lumped together as classic VIN. This change is to reflect the lack of evidence of progression from VIN 1 to VIN 3 (as in the cervix). However, some cases of VIN 1 with high-risk HPV are known, suggesting VIN 1 could be pre-malignant in nature. Thus, there has also been a proposal to have a two-tier clarification with low-grade VIN encompassing condylomas and VIN 1 and high-grade VIN encompassing VIN 2 and 3 and also differentiated VIN. 


\section{$\underline{\text { Microinvasion in borderline serous ovarian tumours }}$}

Is this a risk lesion? The conventional wisdom suggests that it is not but recent data has shown that microinvasion is an adverse prognostic factor independent of stage of disease and implant status in non-pregnant patients (71\% v 97\% 5 year survival)

\section{The implications of the 2009 FIGO staging system for endometrial and vulval malignancies}

The introduction of lymph node status in endometrial malignancies has meant additional specimens to handle but at the same time the problem of false positive peritoneal fluid cytology has been resolved. Assessment of cervical involvement by the endometrial malignancy has become easier.

In vulval cancer, there is more detailed analysis of lymph node involvement required with the introduction of substages in stage 3. One now needs to asses how many lymph nodes are involved and what the size of the metastasis is.

For the first time there are now FIGO stages available for the uterine sarcomas rendering it easier to assign the appropriate stages based on size, etc.

\section{Literature}

1. Broaddus RR, Lynch HT, Chen LM, et al. Pathological features of endometrial carcinoma associated with HNPCC: A comparison with sporadic endometrial carcinoma. Cancer 2006; 106(1): 87-94.

2. Meyer LA, Broaddus RR, Lu LH. Endometrial cancer and Lynch Syndrome: Clinical and pathologic considerations cancer control, 2009; 16(1): 14-22.

3. WHO classification of tumours: Pathology and Genetics of Tumours of the Breast and Female Genital Organs: Tumours of the uterine corpus. IARC Press; 2003: 228-233.

4. Bergeron C, Nogales F, Masseroli M, et al. A multicentric European study testing the reproducibility of the WHO classification of endometrial hyperplasia with a proposal of a simplified working classification for biopsy and curettage specimens. Am J Surg Path 1999; 23: 1102-1108.

5. Mutter GL. Diagnosis of premalignant endometrial disease. J Clin Path, 2002; 55: 326-331.

6. FIGO Committee on Gynaecologic Oncology. Int J Gynaecol \& Obs 2009; 105: 103-104.

7. Carlson JW \& Mutter GL. Endometrial intraepithelial neoplasia is associated with polyps and frequently has metaplastic change. Histopathology, 2008; 53: 325-332.

8. Zhang W, Liong SX, Yu X, et al. Occurrence of endometrial glandular dysplasia precedes uterine papillary serous carcinoma. Int J Gynaecol Pathol 2007; 26: 38-52.

9. Miltel K \& Da Costa D. Endometrial hyperplasia and carcinoma in endometrial polyps: Clinicopathologic and follow-up findings. Int J Gynaecol Pathol 2008; 27: 45-48.

10. Hui P et al. Minimal uterine serous carcinoma: a clinicopathological study of 40 cases. Mod Pathol 2005; 18: 75-82

11. Sherman ME, Ronnett BM, Ioffe OB, et al. Reproducibility of biopsy diagnosis of endometrial hyperplasia: Evidence supporting a simplified classification. Int J Gynaecol Pathol 2008; 27: 318-325.

12. Mutter, MD. www.endometrium.org

13. Hirschowitz L, Ganesan R and McCluggage WG. WT1, p53 and ER receptor expression in uterine serous carcinoma. Histopathol 2009; 55: 478-82

14. Rabban JT and Zaloudek CJ. Minimal uterine serous carcinoma: current concepts in diagnosis and prognosis. Pathology 2007; 39: 125-33 
15. Longacre TA, McKenney JK, Tazelaar HD et al. Ovarian serous tumours of low malignant potential (borderline tumours): outcome-based study of 276 patients with long-term ( $>$ or $=5$-year) followup. Am J Surg Pathol 2005; 29: $707-723$

16. McKenney JK, Balzer BL, Longacre TA. Patterns of stromal invasion in ovarian serous tumours of low malignant potential (borderline tumours): a reevaluation of the concept of stromal micro-invasion. Am J Surg Pathol 2006; 30: 1209-21

17. Carcangiu ML, Peissel B, Pasini B et al. Incidental carcinomas in prophylactic specimens in BRCA1 and BRCA2 germi-line mutation carriers, with emphasis on fallopian tube lesions: report of 6 cases and review of the literature. Am J Surg Pathol 2006; 30: 1222-30

18. Jarboe EA, Folins AK, Drapkin R et al. Tubal and ovarian pathways to pelvic epithelial cancer: a pathological perspective. Histopathology 2008; 53: 127-38 